\title{
Elderly patients and PD-L1-positive advanced non-small cell lung cancer: is pembrolizumab monotherapy effective and safe?
}

\author{
Cesare Gridelli, Assunta Sgambato \\ Division of Medical Oncology, “S. G. Moscati” Hospital, Avellino, Italy \\ Correspondence to: Cesare Gridelli, MD, Division of Medical Oncology, “S. G. Moscati” Hospital, Avellino, Italy. Email: cgridelli@libero.it. \\ Provenance: This is an invited article commissioned by the Section Editor Dr. Jianrong Zhang (Incoming PhD Candidate, Centre for Cancer \\ Research, Faculty of Medicine, Dentistry and Health Sciences, University of Melbourne; Victorian Comprehensive Cancer Centre, Melbourne, \\ Victoria, Australia). \\ Comment on: Nosaki K, Saka H, Hosomi Y, et al. Safety and efficacy of pembrolizumab monotherapy in elderly patients with PD-L1-positive \\ advanced non-small-cell lung cancer: Pooled analysis from the KEYNOTE-010, KEYNOTE-024, and KEYNOTE-042 studies. Lung Cancer \\ 2019;135:188-95.
}

Submitted Oct 22, 2019. Accepted for publication Nov 18, 2019.

doi: $10.21037 /$ atm.2019.12.31

View this article at: http://dx.doi.org/10.21037/atm.2019.12.31

The administration of drugs acting on the immune system in order to improve the body's ability to recognize and destroy cancer cells is really changing the history of lung cancer. In the pre-immunotherapy era, only $5.5 \%$ of patients with advanced non-small cell lung cancer (NSCLC) were alive 5 years after diagnosis and the majority of these patients did not exceed the year (1). The advent of immunotherapy in clinical practice has significantly impacted survival of these patients, particularly in those expressing $\mathrm{PD}-\mathrm{L} 1 \geq 50 \%$ achieving a 5 -year survival rate of about $30 \%$ with median overall survival (OS) of 35.4 months (2). Although about half of all newly diagnosed NSCLC people are elderly (aged $\geq 65$ years) (1), there are currently limited studies on the efficacy and safety of immunotherapy in this age group due to the underrepresentation of older patients in clinical studies. Progressive organ functional reserve failure (mainly of kidney, liver, hearth and bone-marrow), preexisting comorbidities (such as chronic obstructive pulmonary disease, hypertension, diabetes, history of atrial fibrillation, chronic cardiac ischemia, clinical heart failure, previous stroke) and co-medications that may be contraindicated limit the enrollment of elderly patients in clinical lung cancer trials (3). There have also been concerns that the aged-associated decline in the immune systems (so called "immunosenescence") may theoretically affect the clinical profile of immunotherapy in elderly patients. To date, the potential impact of age on the efficacy and toxicity of immune checkpoint inhibitors is still a matter of debate. In the lack of data from large randomized trials designed specifically for elderly patients, alternative studies (for example expanded access program and retrospective cohort studies) tried to answer the question with conflicting results.

Comparable efficacy of immune-agents in older and younger adults when using an age cutoff of 65 years emerged from a meta-analysis of nine randomized controlled trials, in which patients with NSCLC were treated with nivolumab, pembrolizumab or atezolizumab in comparison with chemotherapy/targeted therapy (4). In a recent pooled analysis, patients aged over 65 years with advanced NSCLC, including those $\geq 75$ years, seemed to derive similar survival benefits from immunotherapy as patients less than 65 years of age. Furthermore, patients 75 and older enrolled appeared to tolerate the treatment reporting lower incidence of grade 3 or 4 AEs compared to the subgroup of patients aged $<65$ years (5). Another systematic review and meta-analysis including 12 randomized clinical trials revealed that immune checkpoint inhibitors can improve OS for patients with advanced lung cancer when compared to controls and the magnitude of benefit in OS had comparable efficacy in both younger and older arms using a cut-off of 65 years. Conversely, older patients failed to acquire benefit from immunotherapy when subdivided with a further cut-off of 75 years (6). Focusing on survival outcomes in predefined age groups, 
nivolumab versus docetaxel achieved a reduction of the risk of death in the subset of patients between the ages 65-75 years of $44 \%$ in CheckMate 017 [hazard ratio (HR) 0.56] and 37\% in CheckMate 057 study (HR 0.63), while it seemed to be less effective than chemotherapy in patients aged 75 years or older (HR 1.76 and 0.90 , respectively). However, no firm conclusions were drawn from these trials due to the small number of patients included within this subgroup $(7,8)$. Confirmatory data on efficacy and safety of nivolumab in pretreated elderly patients came from the Italian expanded access program $(9,10)$. Recent results from two trials of nivolumab (CheckMate 171 and CheckMate 153) that have included previously treated patients aged 70 years or older with advanced NSCLC have both demonstrated a comparable survival outcome between the overall population and elderly patients (estimated 6-month OS rate: $67 \%$ vs. $66 \%$, respectively, in CheckMate 171 ; 1 - and 2 -year OS rates: $43 \% / 26 \%$ vs. $44 \% / 25 \%$, respectively in CheckMate 153) (11,12). Similar proportions of patients experiencing treatment-related adverse events (AEs) were reported (50\% vs. 56\% in CheckMate 171 and $62 \%$ vs. $64 \%$ in CheckMate 153 between overall population and elderly patients, respectively) $(11,12)$. Likewise, atezolizumab achieved a longer OS than docetaxel in pretreated patients with advanced NSCLC under the age of 65 years (HR, 0.80) and those aged 65 years or older (HR, 0.66 ) enrolled in the phase 3 OAK trial (13).

On the other hand, pembrolizumab in comparison with docetaxel (phase 2/3 KEYNOTE-010 trial) significantly improved OS among 1,034 pretreated patients with PD$\mathrm{L} 1$ positive ( $\mathrm{PD}-\mathrm{L} 1 \geq 1 \%$ ) advanced NSCLC younger than 65 years (HR 0.63), while reported a non-significant $24 \%$ reduction in the $65-69$ years group (41\% of the enrolled population; HR 0.76 ). There were no patients older than 70 years (14). In the phase 3 KEYNOTE-024 study, firstline pembrolizumab as monotherapy demonstrated an OS benefit over chemotherapy in 305 untreated patients with PD-L1 tumor proportion score (TPS) of $50 \%$ or greater (median OS: 30.0 vs. 14.2 months with chemotherapy; HR 0.63) (15). A statistically survival benefit with pembrolizumab was seen across all analyzed subgroups, including elderly patients: in the 164 patients over the age of 65 (54\% of the enrolled population) the HR for OS was $0.64(15)$.

Recently, results from KEYNOTE-042 study confirmed and extended those from KEYNOTE-024 by demonstrating significantly improved OS with pembrolizumab versus chemotherapy not only in treatment-naïve patients with
PD-L1 TPS $\geq 50 \%$ (HR 0.69) but also in those with low PD-L1 TPS (PD-L1 TPS $\geq 20 \%$ : HR 0.77; PD-L1 TPS $\geq 1 \%$ : HR 0.81$)$ (16).

To evaluate the efficacy and safety of pembrolizumab in elderly patients, Nosaki et al. performed a pooled analysis including 264 elderly patients ( $\geq 75$ years of age, of which 149 treated with pembrolizumab and 115 with chemotherapy) and 2348 patients of $<75$ years of age with PD-L1-positive advanced NSCLC from the three randomized clinical trials previously described (KEYNOTE-010, KEYNOTE-024 and KEYNOTE-042) (17). All patients had PD-L1 TPS of $1 \%$ or higher and half of the elderly group in this analysis had scores of at least $50 \%$. In overall elderly population (treatment-naive and previously treated patients), pembrolizumab significantly improved median OS compared to chemotherapy (median OS: $15.7 \mathrm{vs}$. 11.7 months, respectively; HR 0.76 ). About $54 \%$ of elderly patients in pembrolizumab arm were still alive at one year of treatment compared to $48 \%$ of those receiving chemotherapy. By comparison, the same HR (HR 0.76) was reported in younger patients with 1 -year OS of $54.9 \%$ and $46.9 \%$ in pembrolizumab and chemotherapy arm, respectively. As expected, the magnitude of benefit with pembrolizumab was greater in elderly patients with higher level of PD-L1 expression (PD-L1 TPS $\geq 50 \%$ median OS: 23.1 vs. 8.3 months in chemotherapy arm, respectively; HR 0.40). By age-groups comparison, older patients with a PD-L1 TPS $\geq 50 \%$ appeared to derive even a greater benefit from pembrolizumab than younger patients: one-year OS rate was $61.7 \%$ in both age groups in comparison to just $30.4 \%$ and $49.1 \%$ among elderly and younger patients treated with chemotherapy, respectively (HR 0.40 and HR 0.67, respectively). Among 93 treatment-naïve elderly patients with a PD-L1 TPS $\geq 50 \%$, pembrolizumab as first-line treatment confirmed the survival benefit compared to chemotherapy (median OS: $27.4 v s .7 .7$ months, respectively; HR, 0.41), similar to younger patients (median OS: 20.0 vs. 13.0 months; HR, $0.71)$. Concerning safety profile, fewer elderly patients treated with pembrolizumab presented treatment-related AEs than those receiving chemotherapy (68.5\% vs. $94.3 \%$ ), as well as, grade $3-5$ AEs $(24.2 \%$ vs. $61 \%)$ and serious treatment-related AEs (16.1\% vs. 26.7\%). Fatigue (17.4\%), decreased appetite and pruritus $(12.8 \%$ each) were the most common AEs related to pembrolizumab treatment in elderly patients. Additionally, comparatively fewer elderly patients discontinued pembrolizumab due to treatmentrelated AEs versus chemotherapy (10.7\% vs. $15.2 \%)$. These 
results were comparable for younger patients. In the elderly group, pembrolizumab treatment was associated with higher incidence of immune-mediated AEs and infusion reactions (24.8\% vs. $6.77 \%$ ) compared to chemotherapy, however there was no difference with younger patients $(25 \%$ vs. $5.9 \%$ ). Overall, pembrolizumab provided a benefit in terms of survival and safety in elderly patients compared to chemotherapy. This finding is consistent with the outcomes observed in the overall study populations in each of the three individual studies.

In conclusion, these data support the use of pembrolizumab monotherapy in elderly patients ( $\geq 75$ years) with advanced NSCLC tumors expressing PD-L1. However, since the data were analyzed post hoc, the retrospective and exploratory nature of this analysis represents a potential limitation. First, notable differences were among the three studies evaluated, such as the different populations included (treatment-naïve and pre-treated, PD-L1 TPS $\geq 1 \%$ or $\geq 50 \%$ ) and the different chemotherapy regimens. Nonetheless, in order to reduce these limitations, outcomes were evaluated in each subgroup (TPS $\geq 1 \%$ or $\geq 50 \%$ ) and particularly in treatment-naïve patients with TPS $\geq 50 \%$. It is important highlight that outcomes observed in these analyses are consistent with those observed in the overall pooled population and comparable with the individual study populations. Regarding differences in chemotherapy regimens, the authors underlined that the survival benefit with pembrolizumab treatment was greater regardless the comparators in each individual study, and safety profile of each chemotherapy regimen was consistent with historical data. Second, the individual trials did not stratified population according to age due to low accrual of elderly patients leading to a great difference in the total number of elderly and younger patients evaluated. However, this imbalance involved both treatment arms and should not affect the results. Finally, older patients included in the joint analysis represent a sample of relatively healthy elderly patients, since all enrolled patients had to meet the inclusion for each of individual clinical trials. Based on these results, selected patients aged $\geq 75$ years with good performance status (ECOG PS $0-1$ ) and no conditions or comorbidities preventing study enrollment are eligible for immunotherapy; however more information are needed to establish its role in a real-world elderly population (17).

While this joint analysis showed no differences about the role of immunotherapy according to age, recent results from a real-world study were a wake-up call that potentially suggested lower efficacy of immune-agents in elderly patients with advanced NSCLC. In this retrospective study, worse survival outcomes have been reported in elderly patients ( $\geq 70$ years) when treated with immunotherapy than younger patients (median OS: 5.5 vs. 13 months, HR 3.86; median progression-free survival: 1.8 vs. 3.6 months, HR 2.10) (18). However, the expression of PD-L1 was known only in $50 \%$ of the patients included, the sample size was small (98 patients evaluated of which 27 aged $\geq 70$ years) and data were retrospectively collected. Furthermore, it should be considered the data collected in real studies were not controlled as accurately as in randomized trials. Nonetheless, in line with the findings of Nosaki et al., this study found that increased age was not associated with a higher rate of immune-mediated AEs (18).

Based on published data, immunocheckpoint inhibitors seem to show a comparable efficacy and safety profiles in older and younger patients. Therefore, age should not to be a deterrent to immunotherapy but particular attention should be pay to potential toxicity, especially in nonfit older adults. Looking to the future, larger prospective studies or broader real-world studies are needed to confirm this results in a real-world population, in which older adults are less fit than those enrolled on clinical trials.

\section{Acknowledgments}

None.

\section{Footnote}

Conflicts of Interest: Gridelli C: honoraria as advisory board or speaker bureau member for Astra Zeneca, BMS, MSD, Roche. The other author has no conflicts of interest to declare.

Ethical Statement: The authors are accountable for all aspects of the work in ensuring that questions related to the accuracy or integrity of any part of the work are appropriately investigated and resolved.

\section{References}

1. Siegel RL, Miller KD, Jemal A. Cancer statistics, 2019. CA Cancer J Clin 2019;69:7-34.

2. Garon EB, Hellmann MD, Rizvi NA, et al. Five-Year Overall Survival for Patients With Advanced Non-SmallCell Lung Cancer Treated With Pembrolizumab: Results From the Phase I KEYNOTE-001 Study. J Clin Oncol 
2019;37:2518-27.

3. Denson AC, Mahipal A. Participation of the elderly population in clinical trials: barriers and solutions. Cancer Control 2014;21:209-14.

4. Elias R, Giobbie-Hurder A, Rahma OE. Efficacy of PD-1 and PD-L1 inhibitors in older adults: a meta-analysis. J Clin Oncol 2017;35:abstr e21544.

5. Marur S, Singh H, Mishra-Kalyani P, et al. FDA analyses of survival in older adults with metastatic non-small cell lung cancer in controlled trials of PD-1/PD-L1 blocking antibodies. Semin Oncol 2018;45:220-5.

6. Zhang L, Sun L, Yu J, et al. Comparison of Immune Checkpoint Inhibitors between Older and Younger Patients with Advanced or Metastatic Lung Cancer: A Systematic Review and Meta-Analysis. Biomed Res Int 2019;2019:9853701.

7. Brahmer J, Reckamp KL, Baas P, et al. Nivolumab versus Docetaxel in Advanced Squamous-Cell Non-Small-Cell Lung Cancer. N Engl J Med 2015;373:123-35.

8. Borghaei H, Paz-Ares L, Horn L, et al. Nivolumab versus Docetaxel in Advanced Nonsquamous Non-Small-Cell Lung Cancer. N Engl J Med 2015;373:1627-39.

9. Grossi F, Crinò L, Catino A, et al. P3.02c-096 Use of Nivolumab in Elderly Patients with Advanced Squamous NSCLC: Results from the Italian Expanded Access Programme (EAP). J Thorac Oncol 2017;12:S1337-8.

10. Migliorino MR, Gelibter A, Grossi F, et al. 1320P Use of nivolumab in elderly patients with advanced nonsquamous NSCLC: Results from the Italian expanded access program (EAP). Ann Oncol 2017;28:460-96.

11. Popat S, Ardizzoni A, Ciuleanu T, et al. 1303PD Nivolumab in previously treated patients with metastatic squamous NSCLC: results of a European single-arm, phase 2 trial (CheckMate 171) including patients aged $\geq 70$ years and with poor performance status. Ann Oncol 2017;28:v460-96.

Cite this article as: Gridelli C, Sgambato A. Elderly patients and PD-L1-positive advanced non-small cell lung cancer: is pembrolizumab monotherapy effective and safe? Ann Transl Med 2019;7(Suppl 8):S282. doi: 10.21037/atm.2019.12.31
12. Spigel DR, McCleod M, Jotte RM, et al. Safety, Efficacy, and Patient-Reported Health-Related Quality of Life and Symptom Burden with Nivolumab in Patients with Advanced Non-Small Cell Lung Cancer, Including Patients Aged 70 Years or Older or with Poor Performance Status (CheckMate 153). J Thorac Oncol 2019;14:1628-39.

13. Rittmeyer A, Barlesi F, Waterkamp D, et a. Atezolizumab versus docetaxel in patients with previously treated non-small-cell lung cancer (OAK): a phase 3, openlabel, multicentre randomised controlled trial. Lancet 2017;389:255-65.

14. Herbst RS, Baas P, Kim D-W, et al: Pembrolizumab versus docetaxel for previously treated, PD-L1-positive, advanced non-small-cell lung cancer (KEYNOTE-010): A randomized controlled trial. Lancet 2016;387:1540-50.

15. Reck M, Rodríguez-Abreu D, Robinson AG, et al. Updated Analysis of KEYNOTE-024: Pembrolizumab Versus Platinum-Based Chemotherapy for Advanced Non-Small-Cell Lung Cancer With PD-L1 Tumor Proportion Score of 50\% or Greater. J Clin Oncol 2019;37:537-46.

16. Mok TSK, Wu YL, Kudaba I, et al. Pembrolizumab versus chemotherapy for previously untreated, PD-L1expressing, locally advanced or metastatic non-small-cell lung cancer (KEYNOTE-042): a randomised, open-label, controlled, phase 3 trial. Lancet 2019;393:1819-30.

17. Nosaki K, Saka H, Hosomi Y, et al. Safety and efficacy of pembrolizumab monotherapy in elderly patients with PDL1-positive advanced non-small-cell lung cancer: Pooled analysis from the KEYNOTE-010, KEYNOTE-024, and KEYNOTE-042 studies. Lung Cancer 2019;135:188-95.

18. Corral de la Fuente E, Barquín García A, Saavedra Serrano C, et al. Abstract 169P_PR. Benefit of immunotherapy (IT) in advanced non-small cell lung cancer (NSCLC) in elderly patients (EP). Ann Oncol 2019;30:ii38-ii68. 